\title{
Adaptive Rate DS-CDMA Systems Using Variable Spreading Factors
}

\author{
Lie-Liang Yang, Senior Member, IEEE, and Lajos Hanzo, Fellow, IEEE
}

\begin{abstract}
In this contribution, adaptive rate transmissions are investigated in the context of direct-sequence code-division multiple-access (DS-CDMA) systems using variable spreading factors (VSFs). In the context of the recently established family of adaptive rate-transmission schemes, the transmission rate is typically adapted in response to the channel's fading-induced quality fluctuation. By contrast, in this contribution the transmission rate is adapted in response to the multiuser interference fluctuations encountered. We present the philosophy of the proposed adaptive rate-transmission scheme and analyze the effective throughput as well as the achievable bit error rate (BER) performance, when communicating over additive white Gaussian noise channels. Our study shows that by employing the proposed VSF-assisted adaptive rate-transmission scheme, the effective throughput may be increased by up to $40 \%$, when compared to that of DS-CDMA systems using constant spreading factors. This increased throughput is achieved without wasting power, without imposing extra interference upon other users, and without increasing the BER.
\end{abstract}

Index Terms-Adaptive rate transmissions, code-division multiple access (CDMA), Markov chain, matched filter receiver, multiuser interference, throughput, variable spreading factors (VSFs).

\section{INTRODUCTION}

D IRECT-SEQUENCE code-division multiple access (DSCDMA) is the prevalent technique in the third-generation (3G) wireless communications systems [1], [2], because it is capable of providing numerous advantages as compared to the other solutions. The capacity of DS-CDMA systems is limited by both the time-varying characteristics of the wireless channel and the multiple-access interference (MAI) or multiuser interference (MUI). The family of efficient techniques designed for compensating for the time-varying nature of the wireless channels include the popular RAKE receiver [3], which is contrived for achieving frequency diversity. Alternatively, multiple transmit and/or receiver antennas can be employed for achieving spatial diversity [4]-[6]. The most efficient technique of combating the MAI is multiuser detection (MUD) [7]. The above techniques have attracted worldwide attention in recent years.

Another efficient technique of increasing the capacity of time-varying wireless channels is the employment of adaptive rate transmissions [8]-[12], in which the transmission rate can be adaptively adjusted according to the instantaneous channel

Manuscript received July 3, 2002; revised June 13, 2003 and October 17, 2003. This work was supported in the framework of the IST Project IST-200134091 SCOUT, which is supported in part by the European Union.

The authors are with the Department of Electronics and Computer Sciences, University of Southampton, Southampton SO17 1BJ, U.K. (e-mail: 1ly@ecs.soton.ac.uk; 1h@ecs.soton.ac.uk).

Digital Object Identifier 10.1109/TVT.2003.822027 conditions. The main philosophy behind adaptive rate transmissions is the real-time balancing of the link budget through adaptive variation of the symbol rate, modulation constellation size and format, spreading factor, coding rate/scheme, etc., or in fact any combination of these parameters. However, the results of [10] and [13] have shown that when a sufficiently high diversity order is available, regardless of whether this is due to transmitter or receiver diversity achieved in the time or frequency domain, the advantages of adaptive rate transmissions erode. Hence, in the context of the 3G DS-CDMA systems using power control, channel fading can be efficiently mitigated by employing both the RAKE receiver and multiple transmitter/receiver antennas. In order to combat the MUI in DS-CDMA systems, as we have mentioned above, the most efficient approach is to use multiuser detection receivers [7]. The main obstacle of employing DS-CDMA MUD receivers is, however, the high complexity of the multiuser detection algorithms. Therefore, the conventional matched-filter-based receiver remains popular because of its simplicity, despite its suboptimal performance.

In this contribution, we consider the problem of how the effective throughput of DS-CDMA systems can be increased when the conventional matched-filter-based receiver is employed. Specifically, we consider the uplink transmission of a single-cell DS-CDMA system, where the number of active mobile users obeys the Poisson distribution [14] and all the signals transmitted by the mobile users are power controlled. Hence, the multiuser interference level can be modeled as a discrete Markov process [14], which describes the number of active mobile users. In order to exploit the time-varying nature of the multiuser interference level, an adaptive rate-transmission scheme using variable spreading factors (VSFs) [15], [16] is proposed for increasing the effective throughput. In contrast to the conventional VSF-assisted adaptive rate-transmission scheme, where the transmission rate is adapted in response to the channel-quality fluctuation recorded at the output of the MUD [17], the transmission rate in the proposed scheme is adapted in response to the time-varying interference level due to the MUI, while maintaining the required target BER value. More explicitly, the mobile users increase their transmission rate when the number of active interfering users decreases, while they decrease their transmission rate in response to an increased number of active interfering users. The number of active interfering users is broadcast to the mobile users by the central base station (BS). In this contribution, the performance of the DS-CDMA systems using the proposed VSF-assisted adaptive rate-transmission scheme is evaluated when communicating over additive white Gaussian noise (AWGN) channels. 


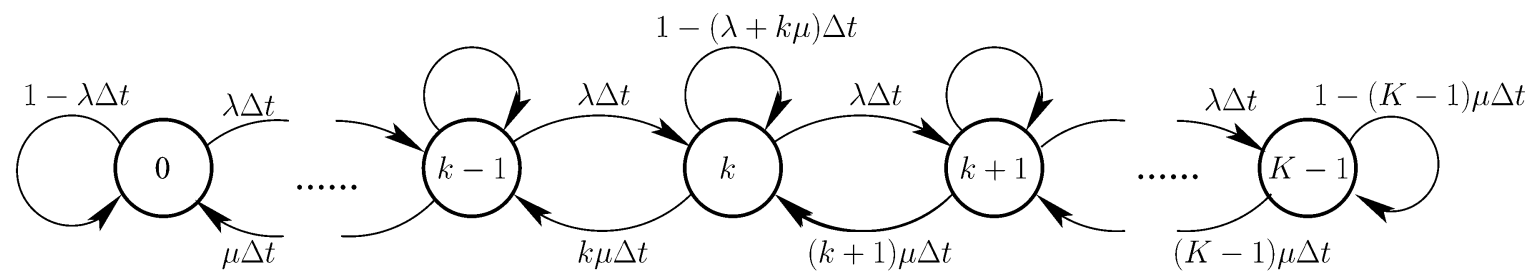

Fig. 1. State-transition diagram modeling the number of active interfering users with the aid of a Markov chain having $K$ states.

The reasons for us to consider only AWGN channels are as follows. First, as we have mentioned above, the fading effects encountered in power-controlled DS-CDMA systems can be efficiently mitigated by using RAKE receivers and multiple transmitter/receiver antennas. Second, our aim is to study the effects of the MUI in isolation, without the obfuscating effects of the channel's fading, and then gain insight into the effects of the MUI on the system's effective throughput when the conventional matched filter receiver is considered. Note that our study can be readily extended for considering various fading channels as well as to multicell CDMA systems. Our results show that by employing VSF-assisted adaptive rate transmissions, the effective throughput of a DS-CDMA system may be increased by $40 \%$ upon exploiting the Markovian-distributed number of active users in the system. The increased effective throughput is achieved without wasting power and without increasing the bit error rate (BER).

The remainder of this contribution is organized as follows. In the next section, we give a rudimentary overview in the context of DS-CDMA systems and introduce the Markov model describing the number of active users, while providing simulation results. In Section III, we describe the VSF-assisted adaptive rate-transmission scheme. Section IV derives the effective throughput of a DS-CDMA system when the proposed adaptive rate-transmission scheme is employed and provides the corresponding BER expression. Our numerical results are provided in Section V and, finally, in Section VI we present our conclusion.

\section{SYSTEM OVERVIEW}

We consider a single-cell DS-CDMA system, where a single BS is located at the center of the cell, while the mobile users are uniformly distributed in the area covered by this BS. The BS is capable of simultaneously processing a maximum number of $K$ calls, i.e., the maximum number of active users supported by the cell is $K$. We assume that each active user's data is BPSK modulated and is transmitted to the BS asynchronously over AWGN channels. Furthermore, we assume ideal power control, i.e., the received power of each active user is the same at the BS. Based on the above assumptions and assuming furthermore that there are $K_{l}+1$ active users (the reference user plus $K_{l}$ interfering users), then the received signal at the BS can be expressed as [18]

$$
r(t)=n(t)+\sum_{k=1}^{K_{l}+1} \sqrt{2 P} a_{k}\left(t-\tau_{k}\right) b_{k}\left(t-\tau_{k}\right) \cos \left(\omega_{c} t+\phi_{k}\right)
$$

where $n(t)$ is the AWGN having a two-sided spectral density of $N_{0} / 2, P$ represents the power received from each active user, $a_{k}(t)$ is the spreading code, $b_{k}(t)$ is the binary data signal, and $\tau_{k}$ is the time-delay parameter that accounts for the propagation delay as well as for the lack of synchronism between the transmitters, while $\phi_{k}$ is the phase angle due to carrier modulation and channel delay.

According to the analysis of [18], the bit errors in DS-CDMA systems communicating over AWGN channels are caused by the effect of multiple-access interference and the AWGN. The BER of an asynchronous DS-CDMA system having received signals given by (1) can be closely approximated by [19]

$$
\begin{aligned}
P_{e}\left(K_{l}\right)= & \frac{2}{3} Q\left[\left(\frac{K_{l}}{3 N}+\frac{1}{2 N \gamma_{c}}\right)^{-1 / 2}\right] \\
& +\frac{1}{6} Q\left[\left(\frac{K_{l} N / 3+\sqrt{3} \sigma}{N^{2}}+\frac{1}{2 N \gamma_{c}}\right)^{-1 / 2}\right] \\
& +\frac{1}{6} Q\left[\left(\frac{K_{l} N / 3-\sqrt{3} \sigma}{N^{2}}+\frac{1}{2 N \gamma_{c}}\right)^{-1 / 2}\right]
\end{aligned}
$$

where $\gamma_{c}=P T_{c} / N_{0}=E_{c} / N_{0}$ represents the signal-to-noise ratio (SNR) per chip and $T_{C}$ is the chip duration, while $N$ represents the spreading factor (number of chips per bit). In our further discourse, $N$ will be controlled as a function of the number of active users and the parameter $\sigma$ can be derived from [19]

$$
\sigma^{2}=K_{l}\left[N^{2} \frac{23}{360}+N\left(\frac{1}{20}+\frac{K_{l}-1}{36}\right)-\frac{1}{20}-\frac{K_{l}-1}{36}\right] .
$$

Furthermore, in (2) the Gaussian $Q$-function is given by $Q(x)=$ $(1 / \sqrt{2 \pi}) \int_{x}^{\infty} \exp \left(-t^{2} / 2\right) d t$.

The number of interfering users $K_{l}$ can be modeled with the aid of a Markov chain having $K$ states [14]. The state-transition diagram modeling the number of users determining the interference level is shown in Fig. 1, which represents a $M / M / m / m$ queueing system [14]. ${ }^{1}$ The arrival rate of new calls or users $\lambda$ corresponds to the probability of the event that a new interfering user is activated within a unit-length time duration, while the average service time of $1 / \mu$ is the average duration of an active interfering connection. For the $M / M / m / m$ queueing system,

${ }^{1}$ In the $M / M / m / m$ queueing system [14], the first parameter $M$ indicates that the arrival process is a Poisson process, the second that $M$ indicates that the service time obeys a negative exponential distribution, the third that $m$ quantifies the number of servers, while the last $(m)$ indicates the limit of the number of customers in the system. 


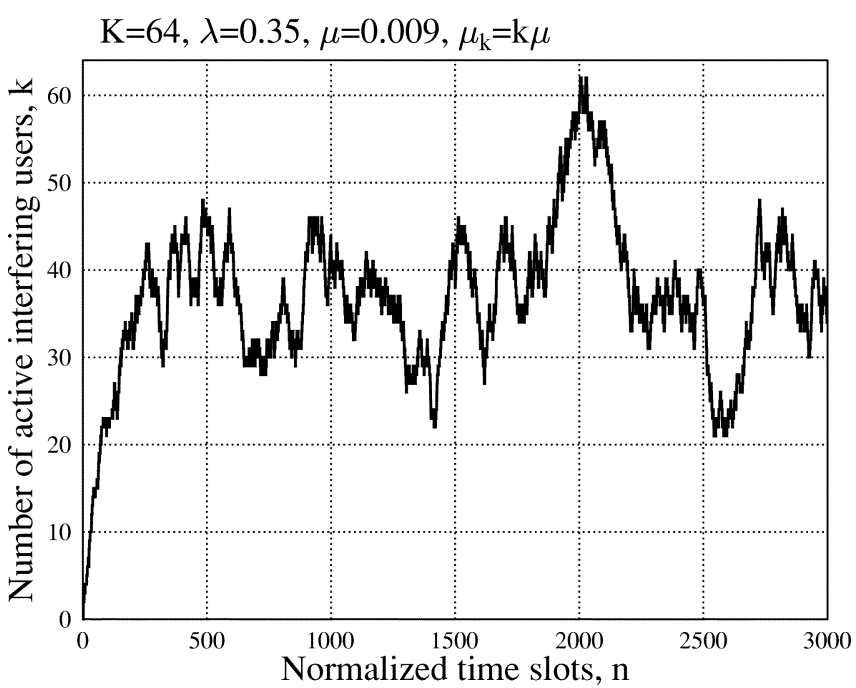

(a)

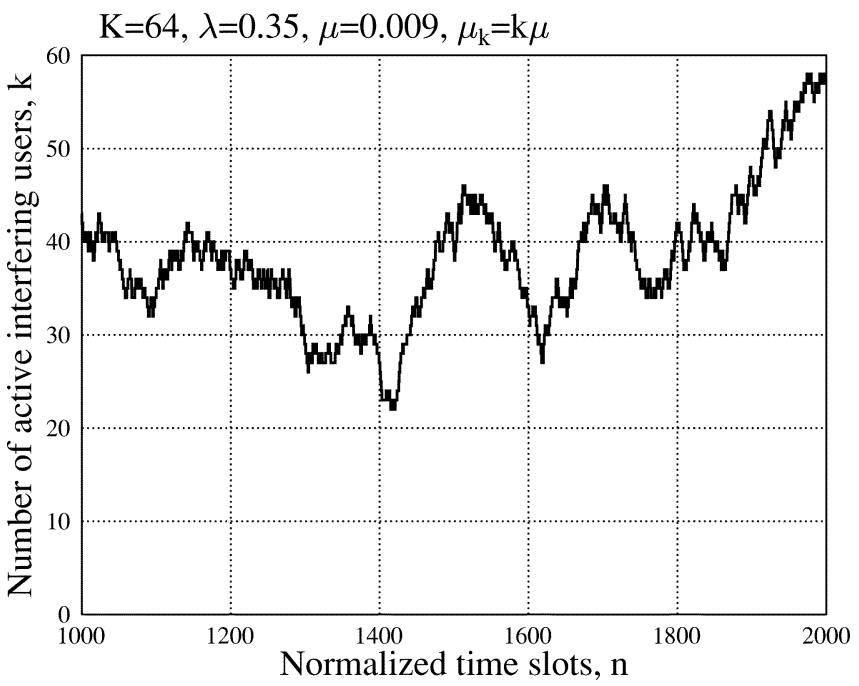

(b)

Fig. 2. Markov characteristics of the number of active interfering users.

the probability that there are $K_{l}$ active interfering users (customers) can be expressed as [14]

$$
p_{K_{l}}=\frac{\left(\frac{\lambda}{\mu}\right)^{K_{l}} \frac{1}{K_{l} !}}{\sum_{m=0}^{K-1}\left(\frac{\lambda}{\mu}\right)^{m} \frac{1}{m !}}, \quad K_{l}=0,1, \ldots, K-1
$$

where the probability of simultaneously supporting $K_{l}=K-1$ users is known as the Erlang $B$ formula [14], which determines the call blocking probability of the system considered.

Fig. 2 shows the number of active users generated by the above-mentioned Markov chain for the first 3000 normalized time slots [Fig. 2(a)] and for the normalized time slots spanning the index-range of 1000-2000 [Fig. 2(b)]. The parameters used for the simulations were $\lambda=0.35, \mu=0.009$, and the maximum number of users supported was $K=64$. From Fig. 2(a) and (b), we can observe that the number of active interfering users is a slowly time-variant variable, fluctuating as a function of the normalized time-slot index.

It is widely recognized that DS-CDMA systems are interference-limited systems and that the systems' BER performance is

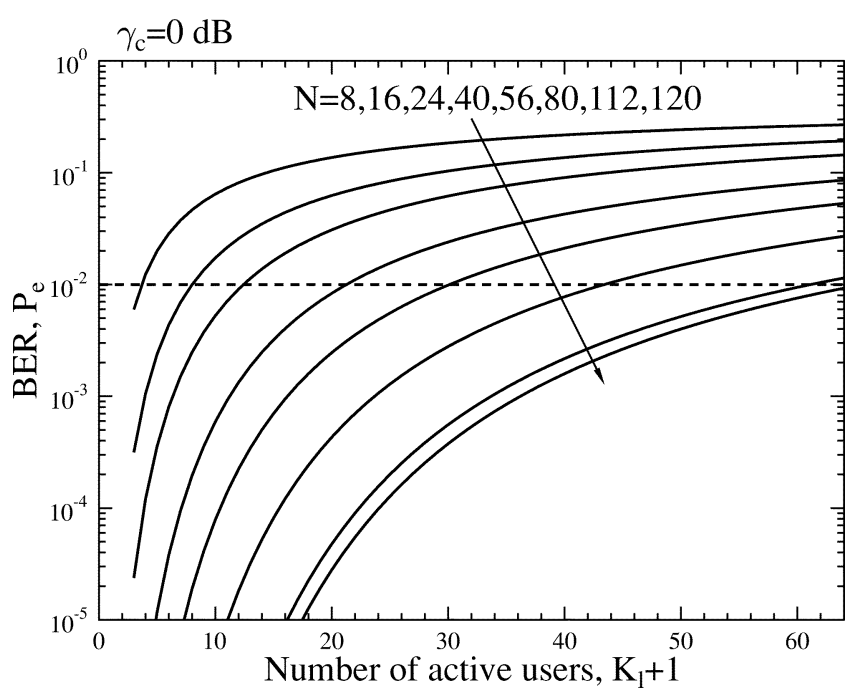

Fig. 3. BER performance versus the number of active users for the parameters of $\gamma_{c}=0 \mathrm{~dB}$ and spreading factors of $N=8,16,24,40,56,80,112$, and 120 computed from (2).

highly sensitive to the number of interfering users. Fig. 3 shows the achievable BER performance with respect to the number of active users for a DS-CDMA system communicating over AWGN channels when the spreading factors of $N=8,16,24$, 40, 56, 80, 112, and 120 are employed. From the results of Fig. 3, we can infer following observations.

- For a given spreading factor, the BER increases when supporting an increased number of active users.

- For a given number of users, the BER decreases upon increasing the value of the spreading factor.

- For a given target BER-for example, for $P_{E}=$ $10^{-2}$ - and for a given number of active users, there exists a spreading factor that results in a specific BER for the DS-CDMA system matching the target BER requirement. A DS-CDMA system using a higher spreading factor is capable of supporting a higher number of active users than that using a lower spreading factor, while maintaining the target BER.

Therefore, based on the Markov chain characterized in Fig. 2 and on the BER performance of the DS-CDMA system as a function of the number of active users shown in Fig. 3, we argue that an appropriate spreading factor can be employed within a specific time slot for maximizing the number of bits transmitted by this specific time slot, while maintaining the required BER performance. Furthermore, when the number of active users dynamically fluctuates, variable spreading factors can be employed by the DS-CDMA system for achieving the maximum throughput, while guaranteeing the required BER performance. In other words, a VSF-assisted adaptive DS-CDMA system is capable of increasing the effective throughput of the system while maintaining a given target BER.

In this section, we have reviewed the behavior of interferencelimited DS-CDMA systems and highlighted the philosophy of an adaptive DS-CDMA system in order to increase the effective throughput of the system when the number of active interfering users is time varying. Let us now investigate the behavior of adaptive VSF-assisted schemes in more detail. 


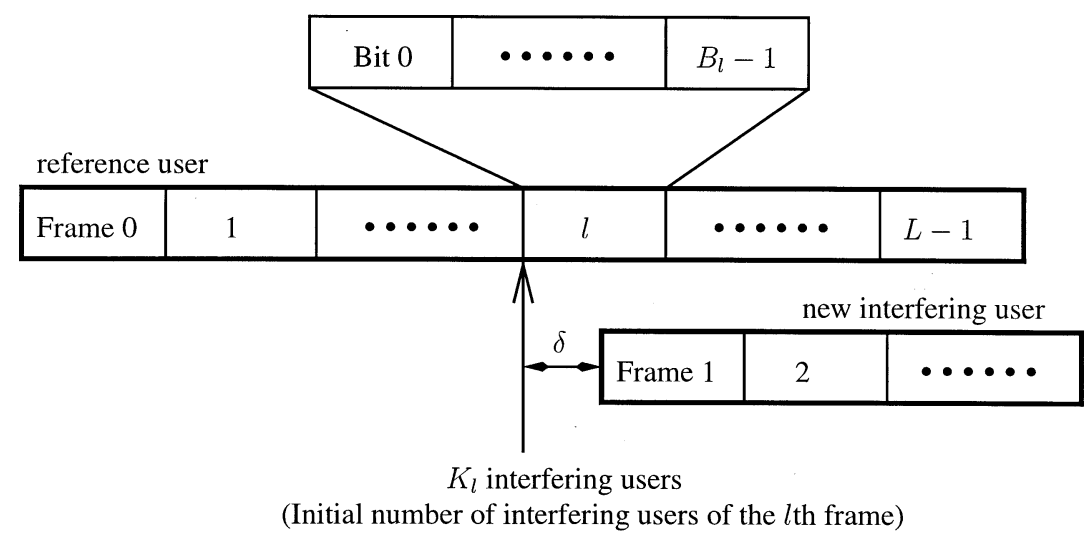

Fig. 4. Data structure of the transmitted signal in adaptive rate DS-CDMA systems using VSF-assisted adaptive rate transmissions.

\section{ADAPTIVE-TRANSMISSION SCHEME}

The requirements for adaptive rate DS-CDMA systems may be listed-without completeness—as follows.

- The rate adaptation of each active user may be controlled independently, i.e., without cooperation with other active users. Hence, the associated complexity is reasonably low.

- Since DS-CDMA systems are typically interference limited, an adaptive rate-transmission scheme must not impose extra interference on the system. Hence, an attractive adaptive rate DS-CDMA scheme is expected to maintain the interference state, regardless of the active users' transmission rates. In other words, an active user's interference environment is expected to be affected only by the number of active users corresponding to a certain level of MUI, but not by their individual transmission rates. The transmission rate and the achievable quality of service (QoS) of a user have to obey a tradeoff for this particular user, regardless of the other users of the system.

- For DS-CDMA systems, where some active users may communicate at constant rates while the remaining active users communicate at a variable rate, the BER and throughput of the active users communicating at constant rates is expected to be unaffected by those communicating using adaptive rate transmissions.

- For adaptive DS-CDMA systems using VSF, the set of legitimate spreading factors must be appropriately designed, so that the effective throughput can be maximized with the aid of readily realizable spreading codes.

Below we propose and investigate a specific adaptive ratetransmission scheme that is capable of meeting the above requirements. Let us assume that each user transmits a block of data, as shown in Fig. 4. As shown in Fig. 4, the data block is divided into $L$ frames, where $L$ is assumed to be a random variable distributed over a certain range, potentially extending to $\infty$. We assume that at the beginning of the $l$ th frame there are $K_{l}$ interfering users, which is the a priori knowledge for determining the required spreading factor, and the corresponding transmission rate during the $l$ th frame. Let us assume that each frame consists of a constant number of chips, which is expressed as $N_{f}$, i.e., each frame has a constant duration of $N_{f} T_{c}$ s. Let us also assume that there are $m$ spreading factors having values expressed as $N_{1}<N_{2}<\ldots<N_{m}$, where each is a factor of $N_{f}$.
Furthermore, let us assume that the target BER is $P_{E}$. Then, the required spreading factor of the $l$ th frame and the corresponding number of bits conveyed by the $l$ th frame can be determined as follows.

- A specific spreading factor is selected from the set $\left\{N_{1}, N_{2}, \ldots, N_{m}\right\}$ according to (2), based on the number of interfering users $K_{l}$ and on the target BER $P_{E}$ such that the selected spreading factor's value is as low as possible while guaranteeing the required BER performance. We denote the selected spreading factor by $N_{l}\left(K_{l}, P_{E}\right)$.

- Once the spreading factor $N_{l}\left(K_{l}, P_{E}\right)$ of the $l$ th frame was selected, the corresponding number of bits $B_{l}\left(K_{l}, P_{E}\right)$ conveyed by the $l$ th frame can be determined as

$$
B_{l}\left(K_{l}, P_{E}\right)=\frac{N_{f}}{N_{l}\left(K_{l}, P_{E}\right)}
$$

Based on (5) and on our previous arguments, it is readily seen that the maximum or minimum throughput of the $l$ th frame is obtained when using the lowest or the highest spreading factor from the set $\left\{N_{1}, N_{2}, \ldots, N_{m}\right\}$, respectively. Specifically, the maximum throughput of the $l$ th frame is given by $B_{l M}\left(K_{l}, P_{E}\right) / N_{f} T_{c}=1 /\left(N_{1} T_{c}\right)($ bits $/ \mathrm{s})=$ $1 / N_{1}$ (bits/chip), while the minimum throughput is given by $B_{l m}\left(K_{l}, P_{E}\right) / N_{f} T_{c}=1 /\left(N_{m} T_{c}\right)($ bits $/ \mathrm{s})=$ $1 / N_{m}$ (bits/chip).

The proposed adaptive-transmission scheme is capable of meeting the requirements listed at the beginning of this section. Specifically, each user's rate adaptation procedure is independent of that of the other users'. The knowledge required by each mobile user for adjusting his or her transmission rate is the number of actively interfering users at the beginning of each transmitted frame. This knowledge can be broadcast to each mobile user by the BS. Second, since each user's received power is constant when different spreading factors as well as different bit durations are used, the interference level is only a function of the number of active interfering users. However, the SNR per bit expressed as $N_{l}\left(K_{l}, P_{E}\right) \gamma_{c}$ exhibits different values when various spreading factors or date rates are employed. Finally, the communication environment of the mobile users employing constant rate transmissions is not 
TABLE I

Number OF BITS TRANSMITTED IN A FRAME BY ASSUMING THAT THE TOTAL Number OF CHIPS PER Frame IS $N_{f}=1680=2^{4} \times 3 \times 5 \times 7$ WHEN THE SPREADING FACTORS SEEN IN THE Right COLUMN ARE EMPLOYED. RANDOM SPREADING CODES WERE ASSUMED

\begin{tabular}{|c|c|c|c|c|c|}
\hline Index & Bits/frame & Spreading factors & Index & Bits/frame & Spreading factors \\
\hline 1 & 1 & 1680 & 21 & 42 & 40 \\
\hline 2 & 2 & 840 & 22 & 48 & 35 \\
\hline 3 & 3 & 560 & 23 & 56 & 30 \\
\hline 4 & 4 & 420 & 24 & 60 & 28 \\
\hline 5 & 5 & 336 & 25 & 70 & 24 \\
\hline 6 & 6 & 280 & 26 & 80 & 21 \\
\hline 7 & 7 & 240 & 27 & 84 & 20 \\
\hline 8 & 8 & 210 & 28 & 105 & 16 \\
\hline 9 & 10 & 168 & 29 & 112 & 15 \\
\hline 10 & 12 & 140 & 30 & 120 & 14 \\
\hline 11 & 14 & 120 & 31 & 140 & 12 \\
\hline 12 & 15 & 112 & 32 & 168 & 10 \\
\hline 13 & 16 & 105 & 33 & 210 & 8 \\
\hline 14 & 20 & 84 & 34 & 240 & 7 \\
\hline 15 & 21 & 80 & 35 & 280 & 6 \\
\hline 16 & 24 & 70 & 36 & 336 & 5 \\
\hline 17 & 28 & 60 & 37 & 420 & 4 \\
\hline 18 & 30 & 56 & 38 & 560 & 3 \\
\hline 19 & 35 & 48 & 39 & 840 & 2 \\
\hline 20 & 40 & 42 & 40 & 1680 & 1 \\
\hline
\end{tabular}

affected by the rate-adaptation operations of the mobile users invoking adaptive rate transmissions.

In [9], Goldsmith and Chua have shown that the highest effective throughput can be achieved by using continuous rate adaptation. Hence, the results of [9] suggest that we should use as many different spreading factors as possible and, simultaneously, have a near-continuous spreading factors value set. Tables I and II show two design examples for the appropriate choice of the spreading factors. In Table I, we assumed that random spreading sequences that have various length were employed and that the frame's length is $N_{f}=2^{4} \times 3 \times 5 \times 7=1680$ chips. As shown in Table I, we can obtain 40 different spreading factors and, hence, can support 40 different transmission rates. In the context of Table II, we assumed that orthogonal spreading sequences were employed and that the frame's length is also $N_{f}=$ $4 \times 2^{2} \times 3 \times 5 \times 7=1680$ chips. These orthogonal spreading sequences having variable length were derived based on the fact that there exist $n \times n(n>2)$ Hadamard matrices, provided that $n=4 t$, where $t$ is a positive integer. Table II shows that there exist 26 different orthogonal spreading codes having different VSF values in conjunction with $N_{f}=1680$. Hence, the DS-CDMA systems using the spreading factors of Table II are capable of supporting 26 different transmission rates.

\section{THROUGHPUT AND BER ANALYSIS}

In this section, we analyze the effective throughput as well as the resultant average BER when achieving this effective
TABLE II

NuMBER OF Bits TRANSMITTED IN A FRAME BY ASSUMING THAT THE TOTAL NuMBER OF CHIPS PER FRAME IS $N_{f}=1680=4 \times 2^{2} \times 3 \times 5 \times 7$ WhEN THE VARIOUS SPREAding FACTORS SEEN IN THE RIGHT COLUMN ARE EMPLOYED. ORTHOGONAL WALSH-HADAMARD SPREADING CODES WERE ASSUMED

\begin{tabular}{c|c|c||c|c|c}
\hline Index & Bits/frame & Spreading factors & Index & Bits/frame & Spreading factors \\
\hline 1 & 1 & 1680 & 14 & 28 & 60 \\
2 & 2 & 840 & 15 & 30 & 56 \\
3 & 3 & 560 & 16 & 35 & 48 \\
4 & 4 & 420 & 17 & 42 & 40 \\
5 & 5 & 336 & 18 & 60 & 28 \\
6 & 6 & 280 & 19 & 70 & 24 \\
7 & 7 & 240 & 20 & 84 & 20 \\
8 & 10 & 168 & 21 & 105 & 16 \\
9 & 12 & 140 & 22 & 140 & 12 \\
10 & 14 & 120 & 23 & 210 & 8 \\
11 & 15 & 112 & 24 & 420 & 4 \\
12 & 20 & 84 & 25 & 840 & 2 \\
13 & 21 & 80 & 26 & 1680 & 1 \\
\hline
\end{tabular}

throughput. The effective throughput is defined as the total number of bits successfully transmitted within a unity-duration time interval by all users supported by the system. Our analysis is based upon the following assumptions.

1) All active users communicate using adaptive rate transmissions based upon the same set of spreading factors, as described in Section III. The transmitted data block length of each active user obeys an independent identical distribution (i.i.d).

2) Assuming that the number of interfering users at the beginning of the $l$ th frame is $K_{l}$, the probability of increasing or decreasing this number by one within a frame's time duration is given by $P_{K_{l}+}=\lambda$ or $P_{K_{l}-}=K_{l} \mu$, respectively. The probability of increasing or decreasing the number of interfering users within a frame's time duration by more than one is zero. Therefore, the probability that the number of interfering users remains unchanged, i.e., $K_{l}$, within a frame's time duration can be expressed as

$$
P_{K_{l}}=\left\{\begin{array}{ll}
1-\lambda, & \text { if } K_{l}=0 \\
1-\lambda-K_{l} \mu, & \text { if } 1 \leq K_{l} \leq K-2 \\
1-K_{l} \mu, & \text { if } K_{l}=K-1
\end{array} .\right.
$$

3) When the number of active interfering users increases by one or decreases by one within the $l$ th frame, we assume that this happens at the moment having a time difference of $\delta$ from the beginning of the $l$ th frame (see Fig. 4), where $\delta$ is assumed to be uniformly distributed over the interval $\left[0, N_{f} T_{c}\right)$.

The effective throughput can be derived as follows. According to our analysis in Sections II and III, we know that the spreading factor $N_{l}\left(K_{l}, P_{E}\right)$ as well as the number of bits $B_{l}\left(K_{l}, P_{E}\right)=N_{f} / N_{l}\left(K_{l}, P_{E}\right)$ transmitted during the $l$ th frame are determined by the number of active interfering users 
at the beginning of the $l$ th frame, as well as by the target BER to be maintained. The effective throughput can be expressed as

$$
\begin{aligned}
B= & E_{\delta}\left[p_{K_{l}=0}\left(\frac{B_{l}\left(0, P_{E}\right)}{N_{f}}+P_{K_{l}+} \times \frac{\left(N_{f} T_{c}-\delta\right) / T_{b}}{N_{f}}\right)\right. \\
& +\sum_{K_{l}=1}^{K-2} p_{K_{l}}\left(\frac{\left(K_{l}+1\right) B_{l}\left(K_{l}, P_{E}\right)}{N_{f}}+P_{K_{l}+}\right. \\
& \left.\times \frac{\left(N_{f} T_{c}-\delta\right) / T_{b}}{N_{f}}-P_{K_{l}-} \times \frac{\left(N_{f} T_{c}-\delta\right) / T_{b}}{N_{f}}\right) \\
& +p_{K_{l}=K-1}\left(\frac{K B_{l}\left(K-1, P_{E}\right)}{N_{f}}-P_{K_{l}-}\right. \\
& \left.\left.\times \frac{\left(N_{f} T_{c}-\delta\right) / T_{b}}{N_{f}}\right)\right]
\end{aligned}
$$

where $T_{b}$ represents the bit duration. Furthermore, in (7) the first term is contributed by the event that there exist no active interfering users. The second term is by the event that there are $K_{l}, K_{l}=1,2, \ldots, K-2$ number of active interfering users, while the last term is contributed by the scenario that there are $K-1$ active interfering users in the system. Since $P_{K_{l}+}=\lambda$ and $P_{K_{l}-}=K_{l} \mu$, (7) can be written as

$$
\begin{aligned}
B= & E_{\delta}\left[\sum _ { K _ { l } = 0 } ^ { K - 1 } p _ { K _ { l } } \left(\frac{\left(K_{l}+1\right) B_{l}\left(K_{l}, P_{E}\right)}{N_{f}}+P_{K_{l}+}\right.\right. \\
& \left.\times \frac{\left(N_{f} T_{c}-\delta\right) / T_{b}}{N_{f}}-P_{K_{l}-} \times \frac{\left(N_{f} T_{c}-\delta\right) / T_{b}}{N_{f}}\right) \\
& \left.-p_{K_{l}=K-1} P_{0+} \times \frac{\left(N_{f} T_{c}-\delta\right) / T_{b}}{N_{f}}\right] \\
= & E_{\delta}\left[\sum _ { K _ { l } = 0 } ^ { K - 1 } p _ { K _ { l } } \left(\frac{\left(K_{l}+1\right) B_{l}\left(K_{l}, P_{E}\right)}{N_{f}}+\lambda\right.\right. \\
& \left.\times \frac{\left(N_{f} T_{c}-\delta\right) / T_{b}}{N_{f}}-K_{l} \mu \times \frac{\left(N_{f} T_{c}-\delta\right) / T_{b}}{N_{f}}\right) \\
& \left.-p_{K_{l}=K-1} \lambda \times \frac{\left(N_{f} T_{c}-\delta\right) / T_{b}}{N_{f}}\right] .
\end{aligned}
$$

Upon taking the expectation with respect to $\delta$, considering that $T_{b}=N_{l}\left(K_{l}, P_{E}\right) T_{c}$ and $B_{l}\left(K_{l}, P_{E}\right)=N_{f} / N_{l}\left(K_{l}, P_{E}\right)$ and substituting $p_{K_{l}}$ from (4) into (7), we finally obtain the effective throughput as

$$
\begin{aligned}
B= & \sum_{K_{l}=0}^{K-1} \frac{\left(\frac{\lambda}{\mu}\right)^{K_{l}} \frac{1}{K_{l} !}}{\sum_{m=0}^{K-1}\left(\frac{\lambda}{\mu}\right)^{m} \frac{1}{m !}}\left[\frac{K_{l}+1}{N_{l}\left(K_{l}, P_{E}\right)}\right. \\
& \left.+\left(\lambda-K_{l} \mu\right) \times \frac{1}{2 N_{l}\left(K_{l}, P_{E}\right)}\right]-\frac{\left(\frac{\lambda}{\mu}\right)^{K-1} \frac{1}{(K-1) !}}{\sum_{m=0}^{K-1}\left(\frac{\lambda}{\mu}\right)^{m} \frac{1}{m !}} \\
& \times \frac{\lambda}{2 N_{l}\left(K-1, P_{E}\right)}[\text { bits/chip]. }
\end{aligned}
$$

In order to derive the average BER, we have to consider the following three events.

Event 1: The number of interfering users during the $l$ th frame remains a constant, namely $K_{l}$. The probability of this event is given by (6) and the average BER is given by $P_{e}\left(K_{l}\right)$ of (2).

Event 2: The number of interfering users increases to $K_{l}+1$ within the $l$ th frame, where the new interfering user commences its transmission $\delta \mathrm{s}$ after the beginning of the $l$ th frame of the reference user, as shown in Fig. 4. The probability of this event is $P_{K_{l}+}=\lambda$ for $K_{l}=0,1, \ldots, K-2$ and $P_{(K-1)+}=0$ for $K_{l}=K-1$. Let $h=\left\lfloor\delta / T_{b}\right\rfloor$, where $\lfloor x\rfloor$ represents the largest integer not exceeding $x$. Then, $\delta$ can be expressed as $\delta=h T_{b}+g$, where $h$ is a discrete random variable uniformly distributed in the interval $\left\{0,1, \ldots, B_{l}\left(K_{l}, P_{E}\right)\right\}$, while $g$ is the continuous random variable uniformly distributed over $\left[0, T_{b}\right)$. Hence, in the context of this event there exist three types of interference patterns within the $l$ th frame. The first interference pattern is encountered in the scenario, that the number of active interfering users associated with the first $h$ number of bits of the $l$ th frame is $K_{l}$. Therefore, the corresponding BER is given by $P_{e}\left(K_{l}\right)$ of (2). The second interference pattern corresponds to the scenario that the number of active interfering users associated with the last $\left[B_{l}\left(K_{l}, P_{E}\right)-h-1\right]$ number of bits of the $l$ th frame is $K_{l}+1$ and, hence, the corresponding BER is given by $P_{e}\left(K_{l}+1\right)$ of (2). The third interference pattern is characteristic of the scenario that there exists a bit in the $l$ th frame, where the leading section of $g=\delta-h T_{b}$ time duration is associated with $K_{l}$ interfering users, while the remaining $T_{b}-g=(h+1) T_{b}-\delta$ time duration is associated with $K_{l}+1$ interfering users. Since $g$ is uniformly distributed in $\left[0, T_{b}\right)$, the average BER associated with this bit can be approximated as $\left[P_{e}\left(K_{l}\right)+P_{e}\left(K_{l}+1\right)\right] / 2$. Consequently, upon considering each of the three above-mentioned interfering patterns, the average BER can be expressed as

$$
\begin{aligned}
P_{e}^{+}\left(K_{l}\right)= & E_{h}\left[\frac { 1 } { B _ { l } ( K _ { l } , P _ { E } ) } \left(h P_{e}\left(K_{l}\right)+\left[B_{l}\left(K_{l}, P_{E}\right)\right.\right.\right. \\
& \left.\left.-h-1] P_{e}\left(K_{l}+1\right)+\frac{P_{e}\left(K_{l}\right)+P_{e}\left(K_{l}+1\right)}{2}\right)\right] \\
= & \frac{1}{\left[B_{l}\left(K_{l}, P_{E}\right)\right]^{2}} \sum_{h=0}^{B_{l}\left(K_{l}, P_{E}\right)-1}\left(h P_{e}\left(K_{l}\right)\right. \\
& +\left[B_{l}\left(K_{l}, P_{E}\right)-h-1\right] P_{e}\left(K_{l}+1\right) \\
& \left.+\frac{P_{e}\left(K_{l}\right)+P_{e}\left(K_{l}+1\right)}{2}\right) \\
= & \frac{1}{2}\left[P_{e}\left(K_{l}\right)+P_{e}\left(K_{l}+1\right)\right] .
\end{aligned}
$$

Event 3: The number of interfering users decreases to $K_{l}-1$ within the $l$ th frame, where one of the active interfering users terminates his or her transmission $\delta \mathrm{s}$ after the beginning of the $l$ th frame. The probability of this event is $P_{K_{l}-}=K_{l} \mu$ for $K_{l}=0,1, \ldots, K-1$. The average BER of Event 3 can be analyzed following the same steps as in the context of Event 2, except that $K_{l}+1$ is now replaced by $K_{l}-1$. The average BER of Event 3 can be expressed as

$$
P_{e}^{-}\left(K_{l}\right)=\frac{1}{2}\left[P_{e}\left(K_{l}\right)+P_{e}\left(K_{l}-1\right)\right]
$$

Finally, by considering the above three possible events, the average BER of the bits in the $l$ th frame can be written as shown in (12) at the bottom of the next page. 
The overall average BER can be computed by averaging $\hat{P}_{e}\left(K_{l}\right)$ with respect to the distribution of the number of active interfering users $K_{l}$, which can be expressed as

$$
\begin{aligned}
P_{e}= & \sum_{K_{l}=0}^{K-1} p_{K_{l}} \hat{P}_{e}\left(K_{l}\right) \\
= & \sum_{K_{l}=0}^{K-1} \frac{\left(\frac{\lambda}{\mu}\right)^{K_{l}} \frac{1}{K_{l} !}}{\sum_{m=0}^{K-1}\left(\frac{\lambda}{\mu}\right)^{m} \frac{1}{m !}} \times\left(\frac{\lambda}{2}\left[P_{e}\left(K_{l}\right)+P_{e}\left(K_{l}+1\right)\right]\right. \\
& \left.+\frac{K_{l} \mu}{2}\left[P_{e}\left(K_{l}\right)+P_{e}\left(K_{l}-1\right)\right]+\left(1-\lambda-K_{l} \mu\right) P_{e}\left(K_{l}\right)\right) \\
& -\frac{\left(\frac{\lambda}{\mu}\right)^{K-1} \frac{1}{(K-1) !}}{\sum_{m=0}^{K-1}\left(\frac{\lambda}{\mu}\right)^{m} \frac{1}{m !}} \times\left(\frac { \lambda } { 2 } \left[P_{e}(K-1)\right.\right. \\
& \left.\left.+P_{e}(K)\right]-\lambda P_{e}(K-1)\right) .
\end{aligned}
$$

Note that in (13), $P_{e}\left(K_{l}\right), P_{e}\left(K_{l}+1\right)$, and $P_{e}\left(K_{l}-1\right)$ are functions of $N_{l}\left(K_{l}, P_{E}\right)$, since the spreading factor assumes different values in response to the time-variant number of active interfering users in the context of the VSF-assisted adaptive rate-transmission scheme.

Above we have derived both the effective throughput formula of a single-cell DS-CDMA system and the resultant BER expression when all the mobile users in the cell transmit using the proposed adaptive rate-transmission scheme. In the following section, we characterize the performance of the DS-CDMA system using VSF-assisted adaptive rate transmissions.

\section{NUMERICAL RESULTS AND DISCUSSION}

In this section, we provide some performance results in order to demonstrate the advantages of VSF-assisted adaptive DS-CDMA systems. We will also compare the throughput performance of the proposed adaptive rate and that of the conventional constant-rate DS-CDMA transmission scheme. The spreading factors employed for adaptive rate transmissions are shown in Table I. Furthermore, in the context of the DS-CDMA system using constant rate transmissions, we assumed that at any given SNR/chip value the specific spreading factor was used, which was capable of maximizing the effective throughput while guaranteeing the target BER performance for the given distribution of the number of interfering users.

Fig. 5 shows the spreading factors required by the DS-CDMA system for achieving the target BER of $P_{E}=0.01$ when supporting a different number of active users ranging from one to 64. The spreading factors were chosen from Table I, each of

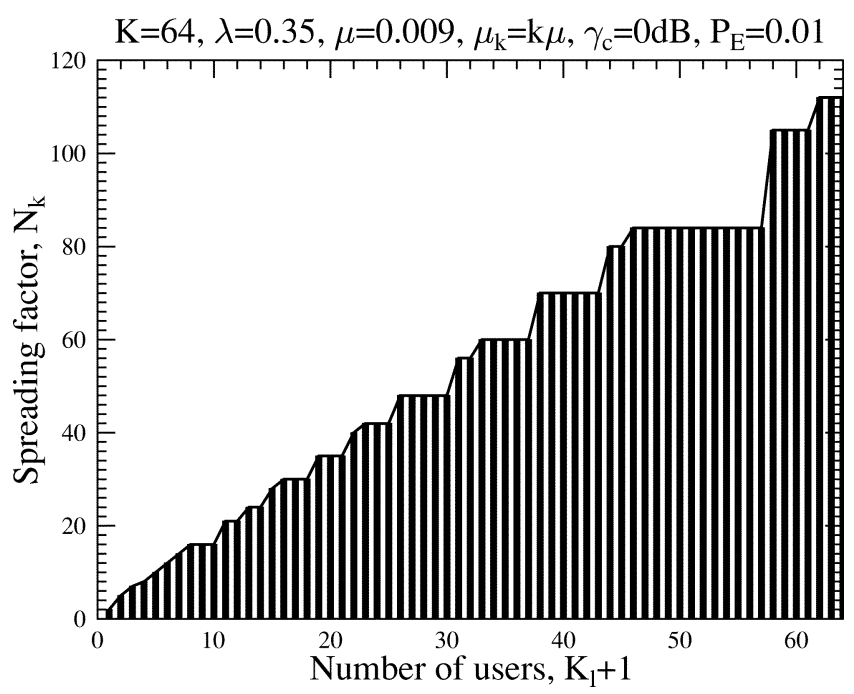

Fig. 5. Spreading factor versus the number of active users required for achieving the target BER of $P_{e}=0.01$.

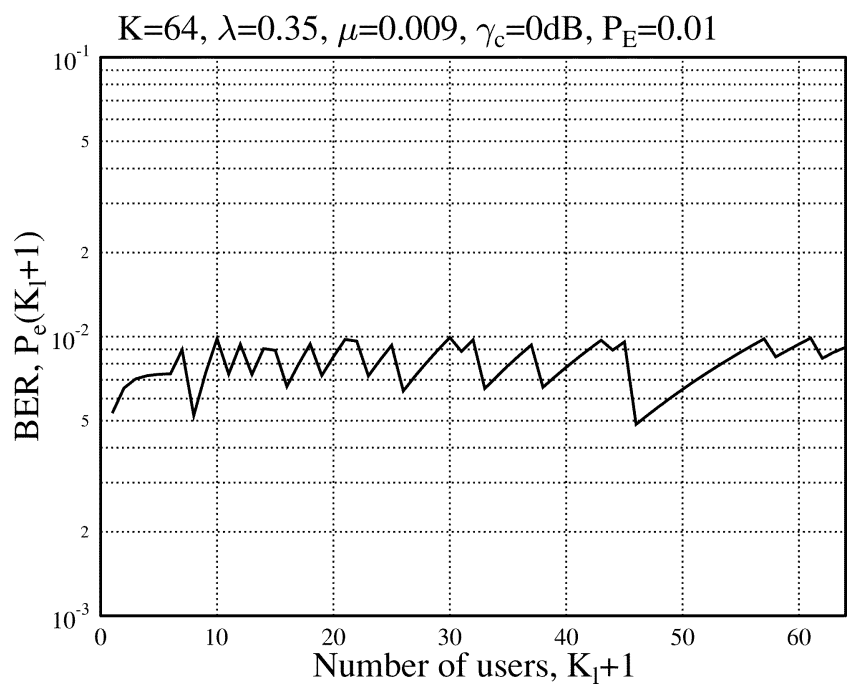

Fig. 6. BER performance versus the number of interfering users when the number of users obeys the distribution of (4), while employing the spreading factors according to Fig. 5.

which guarantee maintaining the target BER for a given number of active users while using the lowest possible spreading factor value. As expected, in order to achieve the required BER, the spreading factor's value has to be increased appropriately, as the number of active users increases.

Fig. 6 shows the resultant BER performance against the number of active users for the DS-CDMA system using the specific variable spreading factors of Fig. 5. The results of Fig. 6 demonstrate that the achieved BER always lies below

$$
\begin{aligned}
\hat{P}_{e}\left(K_{l}\right)= & P_{K_{l}+P_{e}^{+}\left(K_{l}\right)+P_{K_{l}-} P_{e}^{-}\left(K_{l}\right)+P_{K_{l}} P_{e}\left(K_{l}\right)} \\
& = \begin{cases}\frac{\lambda}{2}\left[P_{e}\left(K_{l}\right)+P_{e}\left(K_{l}+1\right)\right]+(1-\lambda) P_{e}\left(K_{l}\right), & \text { if } K_{l}=0 \\
\frac{\lambda}{2}\left[P_{e}\left(K_{l}\right)+P_{e}\left(K_{l}+1\right)\right]+\frac{K_{l} \mu}{2}\left[P_{e}\left(K_{l}\right)+P_{e}\left(K_{l}-1\right)\right] & \\
+\left(1-\lambda-K_{l} \mu\right) P_{e}\left(K_{l}\right), & \text { if } 1 \leq K_{l} \leq K-2 \\
\frac{K_{l} \mu}{2}\left[P_{e}\left(K_{l}\right)+P_{e}\left(K_{l}-1\right)\right]+\left[1-K_{l} \mu\right] P_{e}\left(K_{l}\right), & \text { if } K_{l}=K-1 .\end{cases}
\end{aligned}
$$




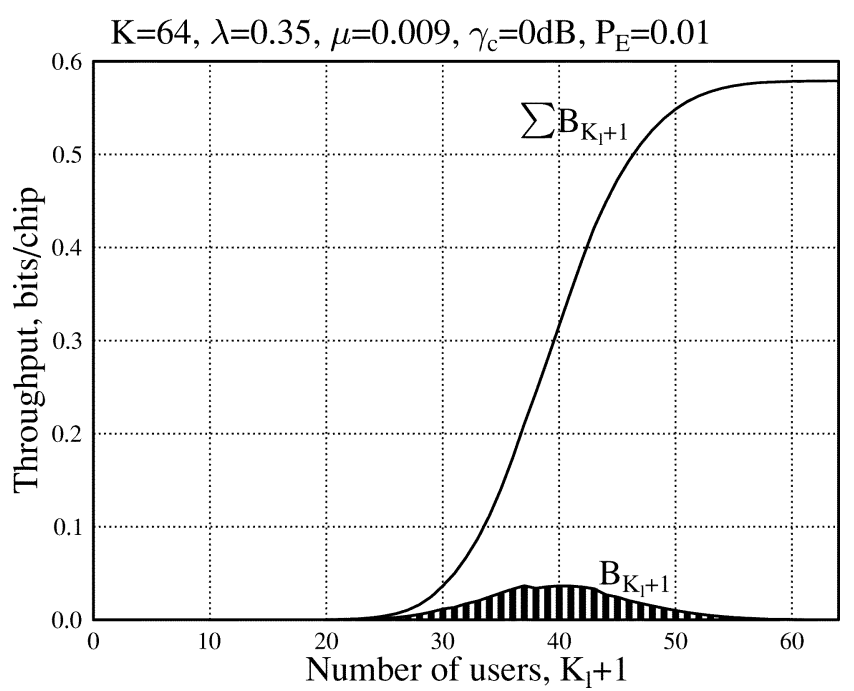

Fig. 7. Throughput density and throughput cumulative functions versus the number of users when the number of interfering users obeys the distribution of (4), while employing the variable spreading factors according to Fig. 5 for the target BER of $P_{e}=0.01$.

the target BER of $P_{E}=0.01$ and is predominantly distributed within the range of $[0.005,0.01)$. A substantial advantage of the associated near-constant BER versus the number of active users is that this results in a significantly improved channel-coding performance in comparison to a fixed-spreading factor-assisted scenario, when the BER fluctuates over a wider range.

Fig. 7 shows the throughput density as well as the throughput cumulative function achieved by the DS-CDMA system when the number of active users obeyed the distribution of (4) for $\lambda=0.35, \mu=0.009$, when the VSFs used assumed the value shown in Fig. 5 and when the DS-CDMA system achieved the BER performance of Fig. 6. In Fig. 7, $B_{K_{l}+1}$ represents the throughput when the number of active users is $\left(K_{l}+1\right)$. As shown in Fig. 7, a large fraction of the effective throughput achieved is contributed when the system supports about 38 active users, which corresponds to the peak of the probability density function (pdf) characterizing the distribution of the number of active users, according to (4). In accordance with the throughput density, the throughput cumulative function increases sharply when the number of active users increases from approximately 30 to approximately 50 . Above this range, it increases slowly and finally reaches the effective throughput of about $0.58 \mathrm{bits} / \mathrm{chip}$. This is the effective throughput provided by the DS-CDMA system at the SNR/chip value of $\gamma_{c}=0 \mathrm{~dB}$, under the experimental conditions used in Figs. 5 and 6.

In Fig. 8, we compared the throughput versus SNR/chip performance of the DS-CDMA system using VSF-assisted adaptive transmissions to that of the DS-CDMA system using constant rate transmissions when various call-generation rates and average call durations are considered. The results related to adaptive rate transmissions were computed from (9) based on the spreading factors of Table I conditioned on the target BER of $P_{E}=0.01$. For the constant rate-transmission scheme, the effective throughput of Fig. 8 recorded at a given SNR/chip value represents the maximum throughput that the DS-CDMA system is capable of achieving. More explicitly, these results were computed as follows. For a given SNR/chip value, we computed

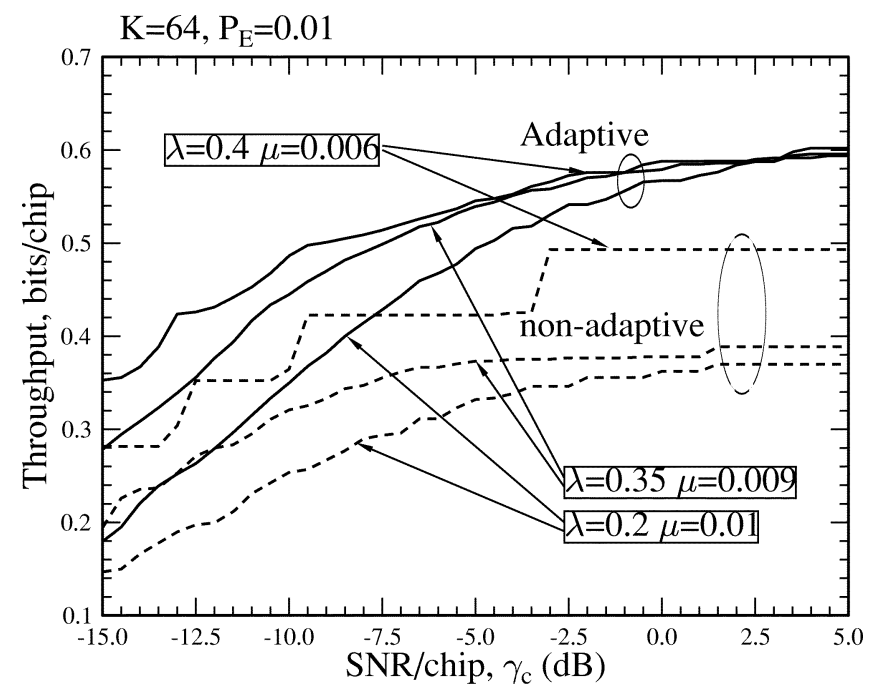

Fig. 8. Throughput performance comparison of the constant spreading factor assisted nonadaptive DS-CDMA scheme and the VSF-assisted adaptive DS-CDMA arrangement.

the corresponding effective throughput results when all possible spreading factors seen in Table I were considered, while taking into account the given distribution of the number of active users as well as the target BER. Then, the maximum of these throughput values was selected as the achievable effective throughput of the constant transmission scheme. The results of Fig. 8 show that the adaptive rate-transmission scheme significantly outperforms the constant rate-transmission scheme. Specifically, the adaptive rate-transmission scheme is capable of providing an approximately $40 \%$ higher effective throughput than the constant rate-transmission scheme. The plausible justification for these results is that when adaptive rate transmission is employed, the system is capable of accommodating the interference level experienced by activating an appropriate spreading factor according to the number of active interfering users at a given SNR/chip value. By contrast, for the constant rate-transmission scheme, only a single spreading factor is employed at a given SNR/chip value, regardless of the number of users supported. Consequently, when the number of active interfering users is low, the BER performance will be better than the target BER and, hence, the effective throughput is correspondingly lower than necessary. However, when the number of active interfering users is excessive, the BER of the received data is higher than the target BER. Hence, the received data may have to be discarded without contributing to the effective throughput.

Finally, in Fig. 9, we show the resultant BER performance of the DS-CDMA system using both constant rate transmissions and the proposed adaptive rate-transmission scheme when they achieve the effective throughput values shown in Fig. 8. From the results of Fig. 9, we observe that for each group of $(\lambda, \mu)$ values, the constant rate scheme has a lower BER than the adaptive rate scheme, while the BER of the adaptive rate-transmission scheme is closer to the target BER than that of the constant rate-transmission scheme. The above observation explains why the adaptive rate-transmission scheme is capable of providing a higher effective throughput than the constant rate-transmission scheme. The results of Fig. 9 demonstrate that neither 


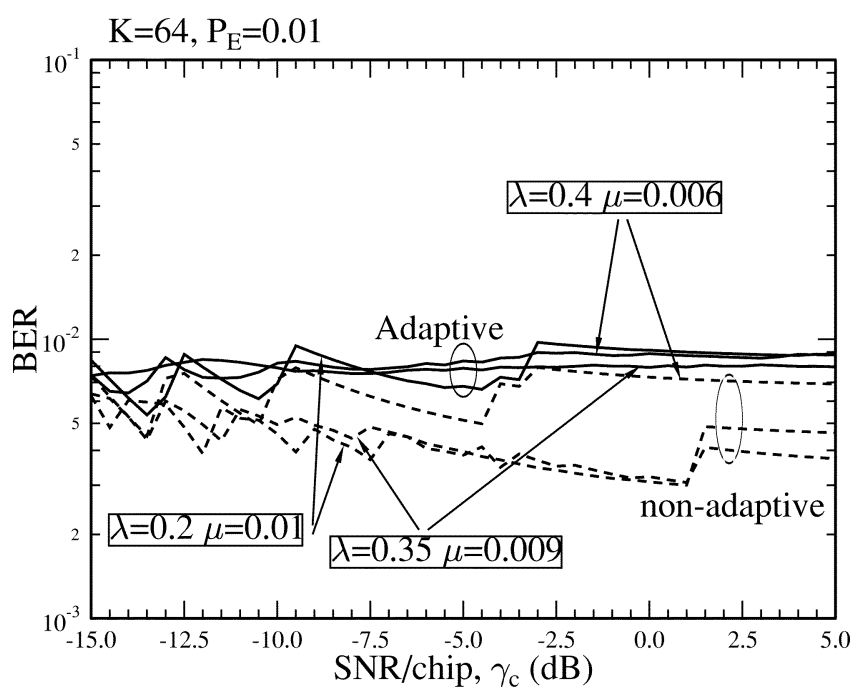

Fig. 9. BER performance comparison between the constant spreading factor assisted nonadaptive DS-CDMA and the VSF-assisted adaptive DS-CDMA schemes when they achieve the effective throughputs of Fig. 8.

the BER of the constant rate nor that of the adaptive rate-transmission scheme fluctuates dramatically. Indeed, they both remain in the BER range of $(0.001,0.01)$ for various SNR/chip values. The reason for observing a near-constant BER even for the constant rate-transmission scheme is because we deliberately adjusted the data rate in response to the SNR/chip value experienced for the sake of fair comparison, although a practical constant-rate system is incapable of doing so. In contrast, the adaptive rate-transmission scheme was capable of adapting the data rate in response to both the SNR/chip value as well as the number of active interfering user supported at each specific SNR/chip value.

\section{CONCLUSION}

In this contribution, we have shown that when the number of active users in a DS-CDMA system is a time-varying random variable and when the conventional matched filter-based receiver is employed, an adaptive rate-transmission scheme using VSFs can be employed for compensating the effects of the time-varying MUI level experienced. The VSF-assisted adaptive rate-transmission scheme is capable of significantly increasing the system's effective throughput. Specifically, our results show that the effective throughput may be increased by up to $40 \%$, when compared to that of DS-CDMA systems using constant spreading factors. This increased throughput is achieved without wasting power, without imposing extra interference upon other users, and without increasing the BER.

Note that the scenario considered in this contribution is a single-cell DS-CDMA system that supports only a single service type. Hence, the work presented in this contribution can be extended by considering multiple-cell DS-CDMA systems that may support a multiplicity of services. For the sake of considering the multiple-cell scenario, each cell can be treated as a Poisson process and adaptive rate transmission is controlled by jointly considering the interference imposed by all active users of the system. Alternatively, adaptive rate transmission can pro- ceed in the same way as described in this contribution, while treating the interference imposed by the adjacent cells as background Gaussian noise. The reason for modeling the interference inflicted by the adjacent cells as Gaussian noise is that the number of interfering users supported by the adjacent cells is relatively high, which results in a reasonable approximation when using the Gaussian interference model.

The extension to the scenario of supporting a multiplicity of services is conceptually straightforward, although the corresponding analysis might be complicated. First, if all services are data based having different data-rate requirements, we have to take into consideration that the interference imposed by a high-rate user is higher than that of a low-rate user. Second, if the services consist of both data and voice, voice users can be treated as constant-rate users, while the data users can use the adaptive rate-transmission regime based on employing VSF. Furthermore, no matter which of the above scenarios is considered, with the aid of adaptive rate transmissions the system's overall throughput can be improved, provided that the number of interfering users or the associated interference level can be modeled as a time-varying variable.

\section{REFERENCES}

[1] L. Hanzo, L.-L. Yang, E.-L. Kuan, and K. Yen, Single- and Multi-Carrier DS-CDMA: Multi-User Detection, Space-Time Spreading, Synchronization, Standards, and Metworking. Piscataway, NJ: IEEE Press-Wiley, 2003.

[2] J. Blogh and L. Hanzo, Third-Generation Systems and Intelligent Wireless Networking: Smart Antennas and Adaptive Modulation. New York: Wiley-IEEE Press, 2002.

[3] G. L. Turin, "Introduction to spread-spectrum antimultipath techniques and their application to urban digital radio," Proc. IEEE, vol. 68, pp. 328-353, Mar. 1980.

[4] P. M. S. Affes, "A new receiver structure for asynchronous CDMA: STAR-The spatio-temporal array-receiver," IEEE J. Select. Areas Commun., vol. 16, pp. 1411-1422, Oct. 1998.

[5] B. Lu and X. D. Wang, "Iterative receivers for multiuser space-time coding systems," IEEE J. Select. Areas Commun., vol. 18, pp. 2322-2335, Nov. 2000

[6] B. Hochwald, T. L. Marzetta, and C. B. Papadias, "A transmitter diversity scheme for wideband CDMA systems based on space-time spreading," IEEE J. Select. Areas Commun., vol. 19, pp. 48-60, Jan. 2001.

[7] S. Verdu, Multiuser Detection. Cambridge, U.K.: Cambridge University Press, 1998.

[8] A. J. Goldsmith and P. P. Varaiya, "Capacity of fading channels with channel side information," IEEE Trans. Inform. Theory, vol. 43, pp. 1986-1992, Nov. 1997.

[9] A. J. Goldsmith and S. G. Chua, "Variable-rate variable-power MQAM for fading channels," IEEE Trans. Commun., vol. 45, pp. 1218-1230, Oct. 1997.

[10] M. S. Alouini and A. J. Goldsmith, "Capacity of Rayleigh-fading channels under different adaptive transmission and diversity-combining techniques," IEEE Trans. Veh. Technol., vol. 48, pp. 1165-1181, July 1999.

[11] V. K. N. Lau, "Variable-rate adaptive channel coding for CDMA-reverse link," Bell Labs. Tech. J., pp. 138-156, Oct./Dec. 2000.

[12] L.-L. Yang and L. Hanzo, "Software defined radio assisted adaptive broadband frequency hopping multicarrier DS-CDMA," IEEE Commun. Mag., vol. 40, pp. 174-183, Mar. 2002.

[13] L. Hanzo, C. H. Wong, and M. S. Yee, Adaptive Wireless Transceivers: Turbo-Coded, Turbo-Equalised and Space-Time Coded Tdma, Cdma, Mc-Cdma and Ofdm Systems. New York: Wiley, 2002.

[14] D. Bertsekas and R. Gallager, Data Networks, 2nd ed. Englewood Cliffs, NJ: Prentice-Hall, 1992.

[15] F. Adachi, M. Sawahashi, and K. Okawa, "Tree-structured generation of orthogonal spreading codes with different lengths for forward link of DS-CDMA mobile radio," Electron. Lett., vol. 33, no. 1, pp. 27-28, 1997. 
[16] F. Adachi, M. Sawahashi, and H. Suda, "Wideband DS-CDMA for nextgeneration mobile communications systems," IEEE Commun. Mag., vol. 36, pp. 56-69, Sept. 1998.

[17] E. L. Kuan and L. Hanzo, "Comparative study of adaptive-rate CDMA transmission employing joint-detection and interference cancellation receivers," in Proc. IEEE VTC'00, Tokyo, Japan, May 2000, pp. 71-75.

[18] M. B. Pursley, "Performance evaluation for phase-coded spread-spectrum multiple-access communication-Part I: System analysis," IEEE Trans. Commun., vol. COM-25, pp. 795-799, Aug. 1977.

[19] J. M. Holtzman, "A simple, accurate method to calculate spread-spectrum multiple-access error probabilities," IEEE Trans. Commun., vol. 40, pp. 461-464, Mar. 1992.

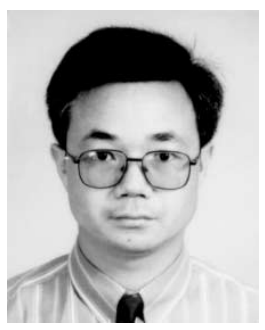

Lie-Liang Yang (M'98-SM'02) received the B.Eng. degree in communications engineering from Shanghai TieDao University, Shanghai, China in 1988 and the M.Eng. and Ph.D. degrees in communications and electronics from Northern Jiaotong University, Beijing, China, in 1991 and 1997, respectively.

From June to December 1997, he was a Visiting Scientist, Institute of Radio and Electronics Engineering, Academy of Sciences, Prague, Czech Republic. Since December 1997, he has been with the Communications Research Group, Department of Electronics and Computer Science, University of Southampton, Southampton, U.K., where he has held various research posts as a Visiting Postdoctoral Research Fellow, Research Fellow, and Senior Research Fellow. Currently, he holds an academic post as a Lecturer. He has been involved in a number of projects funded by the National Sciences Foundations of China, the Grant Agency of the Czech Republic, and the Engineering and Physical Sciences Research Council (EPSRC) of the U.K. and the European Union. He has published over 90 papers in various journals and conference proceedings. His research has covered a wide range of areas in communications, including data network and security, intelligent wireless networking, error-control coding, modulation and demodulation, spread-spectrum communications and multiuser detection, pseudonoise (PN) code synchronization, smart antennas, and adaptive wireless systems, as wel as wide-band, broad-band, and ultrawide-band code-division multiple-access (CDMA) for advanced wireless mobile communication systems.

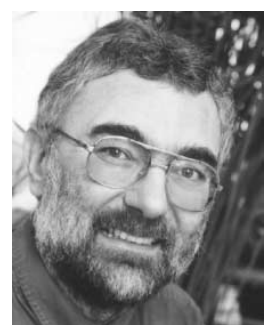

Lajos Hanzo (M'91-SM'92-F'04) received the M.S. and the Ph.D. degrees in electronics from the Technical University of Budapest, Budapest, Hungary, in 1976 and 1983, respectively.

During his 27-year career in telecommunications, he has held various research and academic posts in Hungary, Germany, and the U.K. Since 1986, he has been with the Department of Electronics and Computer Science, University of Southampton, Southampton, U.K., where he holds the chair in telecommunications. Over the years, he coauthored ten Wiley-IEEE Press books on mobile radio communications totaling approximately 8000 pages, published in excess of 450 research papers, organized and chaired conference sessions, presented overview lectures, and has been awarded a number of distinctions. Currently, he is managing an academic research team, working on a range of research projects in the field of wireless multimedia communications sponsored by industry, the Engineering and Physical Sciences Research Council (EPSRC) U.K, the European 6th Framework Programme, and the Mobile Virtual Centre of Excellence (VCE), U.K. He is an enthusiastic supporter of industrial and academic liaison and offers a range of industrial courses. For further information on research in progress and associated publications please refer to http://www-mobile.ecs.soton.ac.uk.

Dr. Hanzo is an IEEE Distinguished Lecturer, as well as a Fellow of the Institution of Electrical Engineers (IEE) 